\title{
A Clinico- Pathological study of Interface Dermatitis
}

\author{
1. Vijaya kumar Hegde 2.Urmila N Khadilkar
}

\begin{abstract}
:
Background: Interface Dermatitis (ID) refers to a pattern of skin reaction characterized by an inflammatory infiltrate that appears to obscure the dermo-epidermal junction when observed at low power examination and referred to as Lichenoid Tissue Reaction (LTR). A wide range of inflammatory skin diseases exhibit interface change with considerable overlap of histological features. The aim of the present study was to study the clinical features and microscopic features of ID

Material and Methods: The material for the present study consisted of skin biopsy samples collected from patients attending the out patient department of dermatology. The study was conducted for a period of three years from 2007 to 2010. During this period a total of 125 cases were studied.

Results : In the present study, a total of 125 cases of Interface Dermatitis (ID) were studied which presented clinically as papulo-squamous disorders. Majority of the cases of ID were seen in women (57.6\%). Majority of ID were Lichen Planus (LP) and its variants (63.2\%). Clinicopathological concordance was seen in 109 cases (87.2\%) and discordance in 16 cases $(12.8 \%)$.

Conclusion: The mere presence of an interface lichenoid inflammatory reaction should not be the sole criterion for the diagnosis of LP or one of its many variants, as now seems to be the case. A clinicopathological correlation is absolutely essential for a conclusive diagnosis of interface dermatitis.
\end{abstract}

Key Words: Interface Dermatitis, Lichenoid disorders, Lichen Planus

\section{Introduction}

Dermatologic diseases characterized by pathology at the dermo-epidermal junction (DEJ) are etiologically diverse and controversial group occurring in a somewhat nebulous anatomical location.

Interface Dermatitis (ID) refers to a pattern of skin reaction characterized by an inflammatory infiltrate that appears to obscure the DEJ when observed at low power examination and referred to as lichenoid tissue reaction (LTR). A wide range of inflammatory skin diseases exhibit interface change with considerable overlap of histological features.

The term 'interface dermatitis' is not used uniformly or consistently. Some apply it to most dermatoses with the LTR. Others use it for the subgroup in which the infiltrate truly obscures the interface (the junction of epidermis and dermis). The term "lichenoid" refers to the papular lesions of certain skin disorders of which Lichen Planus (LP) is the prototype. The papules are shiny, flat topped, polygonal, of different sizes, and occur in clusters creating a pattern that resembles lichen growing on a rock. ${ }^{[1]}$

Although histological study is valuable in diagnosing dermatological disorders, often no definitive diagnosis can be made. A correlation of the interface changes with the clinical diagnosis often helps in arriving at a definitive diagnosis, of the various lichenoid disorders.

\section{Objectives}

1.To determine the histomorphological changes associated with various types of ID

2. To correlate the clinical features with the histological diagnosis.

\section{Material and Methods}

The material for the present study consisted of skin biopsy samples collected from patients attending the out patient department of dermatology

The study was conducted for a period of three years from 2007 to 2010. During this period, a total of 125 cases were studied.

The clinical history and results of other investigations of these cases were taken from the patients' individual case sheets, from the medical records department.

The skin biopsy samples were submitted to the department of Pathology. The biopsy specimens were then fixed in formalin for 24 hours and then processed by routine paraffin-section technique and, stained with Hematoxylin and Eosin. Special stains were done wherever required. All the slides were examined under the light microscopy for epidermal and dermal changes. 


\section{Results:}

In the present study, a total of 125 cases of Interface Dermatitis (ID) were studied which presented clinically as papulo-squamous disorders.

Out of 125 cases studied, the most common type of ID was LP and variants. The second most common being Lichenoid Drug Eruption (LDE) and Lichen Amyloidosis (LA). The least common type of ID was Lichen Nitidus (LN) and Lichen Sclerosus (LS).

ID can affect any age group. Majority of the cases in the present study were in the age range of 41-50 years ( $23.2 \%$ ) followed by $21-30$ years $(21.6 \%)$ and $51-60$ years $(21.6 \%)$ age group. The youngest patient was seven years old and the oldest patient was 70 years of age.

Majority of the cases of ID were seen in women (57.6\%). A female predominance was seen in all cases except in Lichen Simplex Chronicus (LSC) where M:F=2:1. LS affected only males. Equal sex incidence was observed in LA and LN.

Clinicopathological concordance was seen in 109 cases (87.2\%) and discordance in 16 cases (12.8\%). Hundred percent concordance was seen in LA, LS and Erythema Multiforme (EM) because of the classical clinical presentation.

\section{Discussion}

Dermatopathologists are often confronted with tissue sections that show vacuolar degeneration of the basilar layer, necrotic keratinocyte, and upper dermal infiltrate of varying intensity. These components which represent a lichenoid tissue reaction and the variations in the histologic picture have led to the development of the more inclusive term of interface dermatitis for these lesions. ${ }^{[2]}$

At a minimum, a variable combination of leukocyte infiltration of the dermis, vacuolar change of the basilar epidermis, accumulation of melanophages in the upper dermis, and necrosis of keratinocyte must be observed for a diagnosis of interface dermatitis (ID).$^{[3]}$

In the present study, 125 cases of ID were analyzed in which vacuolar change/ degeneration of the basilar epidermis was the most consistent and uniform histologic finding, followed by variable inflammatory infiltrate and pigment incontinence in the dermis. Necrotic keratinocytes were inconsistently seen.

The commonest type of ID observed in the present study was LP. The predominant types of ID other than LP were LA, LDE, LSC and PL. The less common types of ID observed were EM, LN and LS.

ID can affect any age group. In the present study, the age ranged from seven to 70 years; majority of cases were in the age group of 41-50 years (23.2\%) followed by 21-30 years (21.6\%) and 51-60 years (21.6\%). Female preponderance was seen $(57.6 \%)$ among all cases of ID with the ratio being $\mathrm{M}: \mathrm{F}=1: 1.3$

The clinical manifestations of ID is variable. Bereston studied two-hundred cases of lichenoid dermatitis among the SouthWest Pacific War personnel. ${ }^{[4]}$ Three distinct clinical types of lesions were observed: Eczematoid, LPlike and mixed types.

In the present study, out of 125 cases of ID, papulosquamous lesions were predominant (97.6\%). There was no preceding eczematous phase in any of the IDs.

Although LP is the prototype of the lichenoid eruption, many disease processes can produce a lichenoid dermatitis as part of their various histologic presentations. The primary histologic event appears to be T-cell related liquefactive degeneration of the basal epidermis preceding a sequence of degenerative changes within the epidermis. ${ }^{[5]}$

Lichenoid infiltrates are a manifestation of infection, including active infection, or an idiopathic response to nonviable microbial antigen. The organisms include those with superantigen properties, namely viruses, mycobacterial and treponemal species, and streptococci. ${ }^{[6]}$

In the present study, the epidermal changes included hyperkeratosis, parakeratosis, hypergranulosis, acanthosis and Civatte Bodies (CB)

In LP there is a good evidence that activated $\mathrm{T}$ cells attach to the basal epidermal cells and produce death of these cells (Civatte bodies) by apoptosis. When cell death by apoptosis occurs in normal basal cells only small filamentous bodies are produced, many of which are phagocytosed by macrophages or adjacent basal cells. However, when cells have accumulated cytoplasmic filaments prior to the death of the cells, much larger filamentous bodies result which appear incapable of being phagocytosed. Such bodies are usually extruded into the underlying dermis where they become coated with immunoglobulins, particularly $\operatorname{IgM}$, and are recognized as colloid bodies. ${ }^{[7]}$

Singly scattered apoptotic (Civatte) bodies were seen in 52 cases (41.6\%) of ID

Pinkus (1973) outlined the disruption that occurs to the epidermal melanin unit with resulting pigment incontinence. Although some melanin can be seen in the upper dermis in most cases of the LTR, it is more prominent in drug-induced lesions, in patients showing racial pigmentation, and in those lichenoid dermatoses where actinic exposure may play a role. 
In the present study, melanin incontinence was observed in all the cases of ID. It was prominent in all cases of Lichen Planus Pigmentosus (LPP) (100\%) followed by classical LP (75\%) and LSC (44\%).

In the dermis, lymphocytic infiltrate with tendency to invade the lower epidermal layers, capillary proliferation, and participation of histiocytes with formation of pigmented macrophages are characteristic. ${ }^{[8]}$ Extension of cells into surrounding collagen may impart a band-like appearance (or lichenoid appearance, given the similarity to LP) to the infiltrate. Density, pattern, and composition of the infiltrate are variable elements of interface dermatitis that aid in establishing more specific diagnosis.

Interface inflammation was seen in all cases of ID in the present study. The inflammatory infiltrate was composed of exclusively lymphocytes in $71.2 \%$ of cases. In $17.6 \%$ it was lymphohistiocytic. Mixed (lymphocytes, histiocytes, plasma cells and polymorphs) was seen in $11.2 \%$ of cases; eosinophils were present in all cases of LDE and multinucleated giant cells were seen in both the cases of LN.

LA is the commonest type of primary localized cutaneous amyloidosis. Salim, Shenoi and colleagues studied 30 cases of LA. Fifty percent of the patients presented in winter and a genetic predisposition along with winter dryness were considered probable predisposing factors. Histopathologically amyloid deposits were detected in 28 out of 30 patients as uniformly stained pink globules occupying the dermal papilla. Amyloid deposits were visualized using Congo red in all $30(100 \%)$ patients. ${ }^{[9]}$

LA comprised the second most common type of ID in the present study and compared well with regard to gender, incidence, site and amyloid congophilia in the study of Salim et al except that the lesions were predominantly papular and there was no co-existing disorder in any of the cases.

Clinicopathological concordance was seen in 109 cases (87.2\%) and discordance in 16 cases (12.8\%). Hundred percent concordance was seen in LA, LS and EM because of the classical clinical presentation.

The classical histopathological features, together with the clinical information facilitated a better approach to the diagnosis of the various types of ID .

\section{Conclusion}

The present study comprised of 125 cases of papulosquamous disorders of skin, clinically diagnosed as lichenoid dermatitis / interface dermatitis (ID). Histologically, dermoepidermal (interface) change was the mainstay in the diagnosis of ID.

The sequence of antigenic and molecular events that occur in the evolution of lichenoid tissue reaction are responsible for the development of distinctive and specific features such as apoptosis. This helps the dermatopathologists to evaluate the subtle microscopic changes associated with the various types of interface dermatitis. However, it must be emphasized that a clinicopathologic correlation is absolutely essential for a conclusive diagnosis of interface dermatitis. Understanding the individual histopathologic elements of interface dermatitis is the framework on which to build a detailed description of the specific entities. Considerable variation exists in histologic expression of specific diseases depending on body site sampled, adequacy of the sample, and most important, stage of evolution of the lesion sampled.

The mere presence of an interface lichenoid inflammatory reaction should not be the sole criterion for the diagnosis of LP or one of its many variants, as now seems to be the case. Once the nosologic position of a given patient can be determined, the preferred treatment and the prognosis should be much easier and far more accurate than is now possible.

\section{References}

[1]. Tilly JJ, Drolet BA and Esterly NB. Lichenoid eruptions in children. J Am Acad of Dermatol 2004, 606-24.

[2]. Patterson JW. The spectrum of lichenoid dermatitis. J Cut. Pathol 1991, 67-74.

[3]. Horn TD. Interface dermatitis. In Raymond LB, Crowson AN (eds) : Textbook of dermatopathology, $2^{\text {nd }}$ ed, chapter 3. McGrawHill, 2002: 35-61.

[4]. Bereston ES, Lichenoid dermatitis- observation of two hundred cases from the dermatology section, medical branch, dewitt general hospital, auburn California, J of investigative dermatology 1946: 69-83.

[5]. Oliver GF, Winkelmann RK and Muller SA. Lichenoid dermatitis: A clinicopathologic and immunopathologic review of sixty-two cases. J Am Acad of Dermatol 1989: 284-92.

[6]. Magra CM and Crowson AN. Lichenoid and granulomatous dermatitis. Int J of Dermatol 2000: 126-33.

[7]. Weedon D. The lichenoid tissue reaction. Int J of dermatol 1982: 203-6.

[8]. Pinkus H. Lichenoid Tissue Reactions. A speculative review of the clinical spectrum of epidermal basal cell damage with special reference to erythema dyschromicum perstans. Arch Dermatol 1973: 840-46.

[9]. Salim T, Shenoi SD, Balachandran C, Mehta VR .Lichen Amyloidosis .Ind J Dermatol Venerol Leprol; 2005: 166-9. 